Southeast Europe Journal of Soft Computing Volume 1. Number 1 March 2012

\title{
A Fuzzy Model for A Multistage Supply Chain System Controlled By Kanban.
}

\author{
Alp Kor , Mehmet Can \\ Faculty of Economics and Business Administration, \\ International University of Sarajevo, Paromliska 66, Sarajevo, BiH. \\ akor@ius.edu.ba \\ Faculty of Arts and Social Sciences, \\ International University of Sarajevo, Paromliska 66, Sarajevo, BiH. \\ mcan@ius.edu.ba
}

\begin{abstract}
This paper deals with a multi-machine supply chain system operating under just-intime delivery policy with fuzzy demands. For this purpose, the system is modeled as a fuzzy linear programming problem. In JIT delivery policy, kanban has the role of a transporter. By the solution of the related fuzzy linear programming problem, the number of kanbans is determined optimally. Fuzzy demands method enables one to minimize inventory costs under uncertain demand fluctuations. Copyright (C) 2007 IFAC
\end{abstract}

Keywords: Economic and business systems; fuzzy demand; kanban; just in time; inventory cost

\section{INTRODUCTION}

In fuzzy decision making problems, the concept of maximizing decision was proposed by several authors (Bellman et al., 1970). This concept was also adapted to problems of mathematical programming (Tanaka et al., 1984b). Zimmermann (Zimmermann 1983) presented a fuzzy approach to multiobjective linear programming problems. He also studied the duality relations in fuzzy linear programming. Fuzzy linear programming problem with fuzzy coefficients was formulated (Negoita 1970) and called robust programming. Dubois and Prade (Dubois et al., 1982) investigated linear fuzzy constraints. Tanaka and Asai (Tanaka et al., 1984a) also proposed a formulation of fuzzy linear programming with fuzzy constraints and gave a method for its solution which bases on inequality relations between fuzzy numbers. Shaocheng (Shaocheng 1994) considered the fuzzy linear programming problem with fuzzy constraints and defuzificated it by first determining an upper bound for the objective function. Further he solved the soobtained crisp problem by the fuzzy decisive set method introduced by Sakawa and Yana (Sakawa et al.,1985).

In this paper, solution methods of Asai and Shaocheng are combined. The problem is modeled as a fuzzy linear programming 
problem with fuzzy right-hand sides in their constraints. Problem first converted into an equivalent crisp problem. This is a problem of finding a point which satisfies the constraints and the goal with the maximum degree. The idea of this approach is due to Bellman and Zadeh. For solving these crisp linear programming problems the mathematical optimization package LINGO 10.0 is used.

\section{METHOD OF KANBANS}

In the work of Husseini (Husseini et al., 2006) a just in time (JIT) based production system composed of I machines, serially connected to produce a single final product is considered. Each machine consists of a buffer for input material, a manufacturing process and a finished product buffer. Coordination between several machines is achieved using a kanban method.

There are two kinds of kanbans: production kanbans and withdrawal kanbans. Production kanbans are attached to the buffer stock at the inventory point after each production process. This kanban, when the product or part is withdrawn by the downstream machine, is removed and then used for production ordering and the movement of raw materials or parts to the production process. When the part is used for the production, the withdrawal kanban attached to the part is removed and used for the withdrawal of the part to the inventory point.

It is assumed that orders for final product as well as withdrawals from any machine to follow a fixed interval discipline. It is also assumed that finished products delivery from final machine and withdrawal of input material at any machine of production is carried out right before the start of each production period.

\section{METHOD OF FUZZY DEMANDS}

In the following, a model to minimize the inventory cost under fuzzy market conditions is proposed. The numbers of kanbans at each machine of production and at each period are fixed. For purpose of simplicity, container capacity for each product at any stage has been considered as the inventory unit for that item. Table 1 and Table 2 specify the parameters and variables.

Table 1. Parameters used in the fuzzy demand problem.

\begin{tabular}{ll} 
Parameters & Description \\
\hline$I$ & Production stages \\
$T$ & Production periods \\
$C H_{i}$ & Holding cost \\
$C S_{i}$ & Shortage cost \\
$B_{i}$ & $\begin{array}{l}\text { Available production } \\
\text { capacity }\end{array}$ \\
$a_{i}$ & $\begin{array}{l}\text { Required capacity per } \\
\text { product }\end{array}$ \\
$r_{i}$ & Units of input product \\
$U_{i, 0}$ & required per product \\
$\tilde{D}_{t}$ & Initial inventory at the \\
stage $i$
\end{tabular}

Table 2. Variables used in the fuzzy demand problem.

\begin{tabular}{ll} 
Variables & Description \\
\hline$X_{i, t}$ & Production quantity \\
$K_{i}$ & Number of kanbans \\
$U_{i, t}$ & Inventory \\
$U^{+}{ }_{i, t}$ & Positive inventory \\
$U^{-}{ }_{i, t}$ & Negative inventory \\
$R I_{i, t}$ & Unit of products \\
$\mathrm{J} 2-\mathrm{J} 6$ & required \\
\hline
\end{tabular}


Southeast Europe Journal of Soft Computing Volume 1. Number 1 March 2012

Fuzzy demands $\tilde{D}_{t}$ are defined as fuzzy numbers as follows:

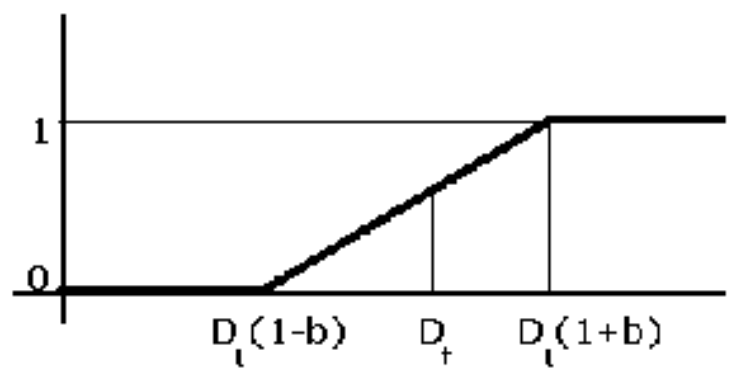

Figure 1. Demands as fuzzy numbers $\tilde{D}_{t}$.

Based on the optimization criteria, the objective is to minimize the function:

$Z=\sum_{(t, i)=(1,1)}^{(T, I)}\left(C H_{i} U^{+}{ }_{i, t}+C S_{i} U^{-}{ }_{i, t}\right)$,

and except for the usage of fuzzy demands, the constraints are as in Husseini et al.,2006:

Production requirement at station $I$, at the period $t$ is equal the number of products required by the market:

$R I_{I, t}=\tilde{D}_{t}, t=1, . ., T$.

Production requirement at station $i$, at the period $t$ is equal to the production at the station $i+1$ times the number of products of station $i$, to produce one product of station $i+1$ :

$R I_{i, t}=r_{i} X_{i+1, t}, t=1, . ., T, i=1, . ., I-1$.

Inventory balance relations are as follows:

$$
\begin{aligned}
& U_{i, 1}=U_{i, 0}+X_{i, 1}-R I_{i, 1}, \quad i=1, . ., I \\
& U_{i, t}=U_{i, t-1}+X_{i, t}-R I_{i, t}, \\
& t=2, . ., T, i=1, . ., I
\end{aligned}
$$

Kanbans at each station $i$, will not be less than the initial inventory:

$$
K_{i} \geq U_{i, 0}, \mathrm{i}=1, \ldots, \mathrm{I}
$$

The number of free kanbans will exceed the production at each station $i$ :

$$
\begin{aligned}
& X_{i, t} \leq K_{i}, \quad \mathrm{t}=1, \ldots, \mathrm{T}, \mathrm{i}=1, \ldots, \mathrm{I}, \\
& X_{i, 1} \leq K_{i}-\mathrm{U}_{\mathrm{i}, 0}, \mathrm{i}=1, \ldots, \mathrm{I}, \\
& X_{i, t} \leq K_{i}-\mathrm{U}_{\mathrm{i}, \mathrm{t}-1}, \\
& \mathrm{t}=2, \ldots, \mathrm{T}, \quad \mathrm{i}=1, \ldots, \mathrm{I},
\end{aligned}
$$

Initial inventory at each station $i-1$ will exceed the demand of production at station $i:$

$$
r_{i-1} X_{i, 1} \leq U_{i-1,0}, \quad i=2, . ., I
$$

Inventory at other times at each station $i-1$ will not exceed the demand of production at station $i$ :

$$
r_{i-1} X_{i, t} \leq U_{i-1, t-1}, \quad t=2, . ., T, i=1, . ., I .
$$

The number production quantity at each station $i$ and at all times will not exceed the production capacity

$a_{i} X_{i, t} \leq B_{i}, \quad t=1, . ., T, i=1, . ., I$.

The number of kanbans and production quantity at each station $i$ will satisfy the following limitations:

$$
\begin{aligned}
& X_{i, 1}-K_{i}+U_{i, 0} \geq M\left(J 2_{i}-1\right), \mathrm{i}=1, \ldots, \mathrm{I}, \\
& X_{i, t}-K_{i}+U_{i, t-1} \geq M\left(J 3_{i, t}-1\right), \\
& \mathrm{t}=2, \ldots, \mathrm{T}, \mathrm{i}=1, \ldots, \mathrm{I}, .
\end{aligned}
$$

End of the period inventory at each station $i-1$ and production quantity at each station $i$ will satisfy the following limitations:

$$
\begin{aligned}
& r_{i-1} X_{i, 1}-U_{i-1,0} \geq M\left(J 4_{i}-1\right), \mathrm{i}=2, \ldots, \mathrm{I}, \\
& r_{i-1} X_{i, t}-U_{i-1, t-1} \geq M\left(J 5_{i, t}-1\right), \\
& \mathrm{t}=2, \ldots, \mathrm{T}, \mathrm{i}=2, \ldots, \mathrm{I}, .
\end{aligned}
$$


Southeast Europe Journal of Soft Computing Volume 1. Number 1 March 2012

Combined with (9), the number production quantity at each station $i$ and at some times will be exactly equal the production capacity:

$$
\begin{aligned}
& a_{i} X_{i, t}-B_{i} \geq M\left(J 6_{i, t}-1\right), \\
& t=1, . ., T, i=1, . ., I
\end{aligned}
$$

Relation between 0,1 switch variables are as follows:

$$
\begin{aligned}
& J 2_{i}+J 4_{i}+J 6_{i, 1}=1, i=2, \ldots, I, \\
& J 3_{i, t}+J 5_{i, t}+J 6_{i, t}=1, \\
& t=2, \ldots, T, i=2, \ldots, I, \\
& J 2_{1}+J 6_{1,1}=1, \\
& J 3_{1, t}+J 6_{1, t}=1, t=2, \ldots, T .
\end{aligned}
$$

Positive and negative situations of the inventory are related as follows:

$U_{i, t}=U^{+}{ }_{i, t}-U^{-}{ }_{i, t}, \quad t=1, . ., T, i=1, . ., I$

One has also the following limitations on variables:

$X_{i, t}, U^{+}{ }_{i, t}, U^{-}{ }_{i, t} \geq 0, U_{i, t}$ unrestricted,

$t=1, \ldots T, i=1, \ldots, I$

$K_{i}$ integer, $i=1, \ldots, I$,

$J 2_{i}, J 3_{i, t}, J 4_{i}, J 5_{i, t}, J 6_{i, t} 0$ or 1 ,

$t=1, \ldots T, i=1, \ldots, I$

Hence the problem is a fuzzy linear programming problem with fuzzy constraints (2) in it.

\section{SOLUTION OF THE KANBAN PROBLEM WITH FUZZY DEMANDS}

For defuzification of this problem, first the objective function is fuzified. This is done by calculating the lower and upper bounds of the optimal values first. The relevant parameter values are taken as follows
Table 3. Parameter values used.

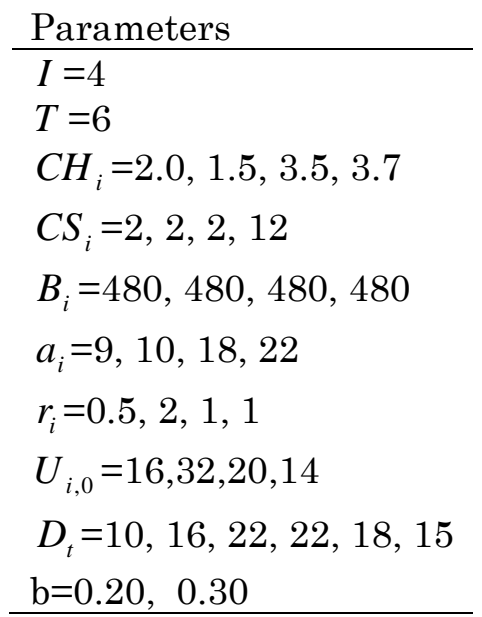

The bounds of the optimal values, $z_{1}$ and $z_{2}$ are obtained by solving the following two standard linear programming problem

$z_{1}=\min \sum_{(t, i)=(1,1)}^{(T, I)}\left(C H_{i} U^{+}{ }_{i, t}+C S_{i} U^{-}{ }_{i, t}\right)$,

under the same restrictions (3-15) and

$$
R I_{I, t}=(1-b) D_{t}, t=1, . ., T \text {. }
$$

and another crisp linear programming problem

$z_{2}=\min \sum_{(t, i)=(1,1)}^{(T, I)}\left(C H_{i} U^{+}{ }_{i, t}+C S_{i} U^{-}{ }_{i, t}\right)$,

under the same restrictions (3-15) and

$$
R I_{I, t}=(1+b) D_{t}, t=1, . ., T .
$$

The objective function takes values between $z_{1}$ and $z_{2}$ while right hand sides in (2) vary between $(1-b) D_{t}$ and $(1+b) D_{t}$. Let $z_{l}=\min \left(z_{1}, z_{2}\right)$ and $z_{u}=\max \left(z_{1}, z_{2}\right)$. Then, $z_{l}$ and $z_{u}$ are called the lower and upper bounds of the optimal values, respectively.

By using the definition of the fuzzy decision proposed by Bellman and Zadeh (Bellman et al., 1970), the optimal fuzzy decision is a solution of the problem 
Southeast Europe Journal of Soft Computing Volume 1. Number 1 March 2012

$\max \lambda$

$\lambda\left(z_{u}-z_{l}\right)+\sum_{(t, i)=(1,1)}^{(T, I)}\left(C H_{i} U^{+}{ }_{i, t}+C S_{i} U^{-}{ }_{i, t}\right)-z_{u} \leq 0$

under all restrictions (3-15), and under the additional constraint

$$
\begin{gathered}
R I_{I, t}=((2 \lambda-1) b+1) D_{t}, \\
t=1, \ldots, T, 0 \leq \lambda \leq 1
\end{gathered}
$$

is solved. For the parameter values in our kanban problem with fuzzy demands the following solutions are obtained for different tolerance values $b$.

Table 4. Upper, lower and compromise solutions.

\begin{tabular}{lllll}
$\mathrm{b}$ & $z_{l}$ & $z_{u}$ & Compromise & Satisfaction \\
\hline 0.20 & 714 & 1314 & 984 & 0.559 \\
0.30 & 696 & 1572 & 1015 & 0.630 \\
\hline
\end{tabular}

Here are the optimal values of decision variables for the compromise solution.

Table 5. Optimal values of decision variables stage 1

\begin{tabular}{llllllll} 
Period & 0 & 1 & 2 & 3 & 4 & 5 & 6 \\
\hline K & 26 & 28 & 28 & 28 & 28 & 28 & 28 \\
RI & & 13 & 12 & 16 & 11 & 16 & 12 \\
X & & 12 & 13 & 11 & 17 & 11 & 16 \\
U & 16 & 15 & 16 & 11 & 17 & 12 & 16 \\
\hline
\end{tabular}

Table 6. Optimal values of decision variables stage 2

\begin{tabular}{llllllll} 
Period & 0 & 1 & 2 & 3 & 4 & 5 & 6 \\
\hline $\mathrm{K}$ & 56 & 58 & 58 & 58 & 58 & 58 & 58 \\
$\mathrm{RI}$ & & 24 & 34 & 20 & 33 & 25 & 33 \\
$\mathrm{X}$ & & 26 & 24 & 32 & 22 & 33 & 23 \\
$\mathrm{U}$ & 32 & 34 & 24 & 36 & 25 & 33 & 23 \\
\hline
\end{tabular}

Table 7. Optimal values of decision variables stage 3

\begin{tabular}{llllllll}
\multicolumn{1}{c}{ Period } & 0 & 1 & 2 & 3 & 4 & 5 & 6 \\
\hline K & 42 & 32 & 32 & 32 & 32 & 32 & 32 \\
RI & & 17 & 10 & 16 & 16 & 16 & 13 \\
X & & 12 & 17 & 10 & 16 & 13 & 16 \\
U & 20 & 15 & 22 & 16 & 16 & 13 & 16 \\
\hline
\end{tabular}

Table 8. Optimal values of decision variables stage 4

\begin{tabular}{llllllll}
\multicolumn{1}{l}{} & 0 & 1 & 2 & 3 & 4 & 5 & 6 \\
Period & & & & & & & \\
\hline K & 22 & 31 & 31 & 31 & 31 & 31 & 31 \\
RI & & 10 & 16 & 22 & 22 & 18 & 15 \\
X & & 17 & 10 & 16 & 16 & 16 & 13 \\
U & 14 & 21 & 15 & 9 & 2 & 0 & -3 \\
\hline
\end{tabular}

\section{CONCLUSION}

In this article, it is shown how to apply fuzzy linear programming to the kanban problem under uncertainties in the demand. The same problem can be handled under other fuzzy restrictions as well. For the risk mitigation in the production line, the most important achievements can be reached through the fuzification of the constraint

$$
R I_{i, t}=r_{i} X_{i+1, t}, t=1, . ., T, i=1, . ., I-1 .
$$

This crisp constraint has no buffer to recover the disruptions and troubles in the production line and in the supply chain. This may be the topic of another research.

The main advantage of the use of the fuzzy linear programming is that it allows us to deal with the fuzzy demands of the market.

\section{REFERENCES}

Bellman, R.E., and L.A. Zadeh (1970). Decisionmaking in a fuzzy environment, Management Science 17 B 141-164.

Dubois, D. and H. Prade (1982). System of linear fuzzy constraints, Fuzzy Sets and Systems 13 1-10.

S.M. Moattar Husseini, C. O'Brien, and S.T. Hosseini

(2006). A Method to enhance volume flexibility in JIT production control, I.J. of Production Economics, 104 653-665. 
Southeast Europe Journal of Soft Computing Volume 1. Number 1 March 2012

Negoita, C.V. (1970). Fuzziness in management, OPSA/

TIMS, Miami .

Sakawa, M., H. Yana (1985). Interactive decision

making for multi-objective linear fractional programming problems with fuzzy parameters, Cybernetics Systems 16 377-397.

Shaocheng, T. (1994). Interval number and Fuzzy

number linear programming, Fuzzy Sets and Systems 66 301-306.

Tanaka, H., and K. Asai (1984a). Fuzzy linear

programming problems with fuzzy numbers, Fuzzy Sets and Systems 131 10.

Tanaka, H., T. Okuda, and K. Asai (1984b).

On fuzzy

mathematical programming, $J$. Cybernetics 3 37-46.

Zimmermann, H.J. (1983). Fuzzy mathematical programming, Comput. \& Ops. Res. 10 No 4 291-298. 